\section{P-138 GROUP EXERCISE WITH INDIVIDUALISED GOALS AND PHYSICAL AND PSYCHOSOCIAL WELLBEING IN PALLIATIVE CARE}

Irene Campagnolo Maschio, Konstantina Chatziargyriou. Princess Alice Hospice, Esher, UK

10.1136/bmjspcare-2017-hospice.163

Background A number of studies have established the benefit of exercise as part of a palliative care programme (Van den Dungen et al., 2014; Salakari et al., 2015; Malcolm et al., 2016; Paltiel et al., 2009), however, there are still research questions to answer about the effects of group exercise interventions.

Aim To assess the impact on the patients' physical and psychosocial wellbeing of an eight week group exercise programme with individualised goals.

Methods We conducted a quantitative and qualitative analysis on data collected from the 19 adult patients included in the study (17 cancer diagnosis, 1 pulmonary fibrosis, 1 neurodegenerative condition). Inclusion criteria: patient willing to participate in a group exercise programme and able to complete the baseline assessment (6 min walk test, Timed up and go, Berg balance scale, EORTC QLQ-C30, HADS, open text questions about their experience of undertaking the intervention).

Results Data suggest an improvement in physical function and minimal changes in psychological function. Returned questionnaires advise that peer and professional support, investment in the patient's goals and a general openness to share and discuss experiences seem to be key elements in the observed positive reframing of the experience of illness and present and future deterioration. We observed increased sense of control, social participation and enjoyment of life.

Conclusions Despite limitations (small sample, no control group), we were able to explore how personalised exercises in a small group can foster patients' resilience possibly through the reappraisal of their condition (Monroe \& Oliviere, 2007) and a reconnection with their own body and experience (Morgan et al., 2017). Interestingly, it was not always straightforward to link data with what was observed clinically and reported by patients e.g., deterioration in tests but improvement in function and general wellbeing. From what was observed, a group exercise intervention in palliative care seems feasible, cost-effective and valid in improving physical and psychosocial wellbeing in the population studied. A control group is to be considered to deepen the analysis.

\section{P-139 STEP UPS AND STICKY BUNS- EVALUATION OF A HOSPICE BASED EXERCISE GROUP}

Sue Cullum. Phyllis Tuckwell Hospice, Farnham, UK

\subsection{6/bmjspcare-2017-hospice.164}

Background Following a literature review and visiting other hospices, it became clear that regular exercise groups can be effective in maintaining fitness and activity levels as well as improving mood and reducing fatigue.

We were keen to ensure that we were meeting the needs of patients with potential to maximise fitness and independence in a way that was good use of time and resources.

Aim To assess the value and effectiveness of hospice-based exercise groups, to inform future planning, ensure best practice and optimise patient experience.
Method Appropriate patients were recruited to a weekly circuit-based exercise group run by a physiotherapist and volunteers. Baseline data were collected and goals set. Reviews took place after a minimum of two months attendance, with assessments repeated, goals reviewed and subjective views collected.

This evaluation was carried out a year after the group was set up.

Results Eight people attended the group regularly, with a variety of terminal diagnoses.

Regular attendance, satisfaction and goal achievement were seen as an indication of effectiveness and value. Improvement in outcome measures were seen $(75 \%$ of two-minute walk tests and $80 \% 5 \times$ sit to stand) but this was considered of secondary importance in this population of patients with deteriorating terminal illness.

Participant feedback underlined the importance of regular supported exercise and goal achievement, resulting in increased confidence, activity and independence as well as reduced reliance on medications. The importance of social support and improved mood was also highlighted.

Lessons were learned that have informed future planning and the setting up of additional exercise groups.

Conclusion Group exercise is an effective way to support people living with a terminal condition, enabling them to remain as active and independent as possible at the same time as providing social support.

\section{P-140 MUSICAL CHAIRS - MORE THAN JUST AN EXERCISE GROUP}

Dympna Jones, Kate Marley, Clare Forshaw, Kate Mclntegart, Helen Cunliffe, Susan Clarkson. Woodlands Hospice, Liverpool, UK

\subsection{6/bmjspcare-2017-hospice. 165}

Background The benefits of exercise for people with life-limiting conditions are widely recognised. Woodlands Hospice runs a weekly exercise group which is well attended. Feedback is positive and the environment provides more than simply physical benefits. It has become a safe place that instils positivity and humour where patients can share feelings and coping strategies, gain support and strength from their peers and staff, ask for help whether physical, emotional or spiritual and where they can just 'be'.

Aim To further develop this group to enhance the experience for patients. Specifically:

- Music. To introduce music in the form of a personal group playlist where all patients are involved in sharing a song and a reason for its choice. This music is played during the group and made available to take home.

- Tai Chi. To teach simple chair based Tai Chi to be practised at the end of each session to promote a calm and contemplative atmosphere.

- Palliative Outcome Scale (POS). Using this recognised tool on a monthly basis helps patients to discuss any new concerns, allows staff to signpost patients appropriately and streamlines outcomes throughout the hospice.

\section{Methods}

- Literature review on clinical benefits of Music therapy and Tai Chi

- Consultation with group members

- Practise Tai Chi sessions 
- Multidisciplinary consultation regarding introduction of POS.

Results

- Music

A feeling of ownership and camaraderie promoting discussion ranging from shared memories to the spiritual needs of the present

- Tai Chi

This new skill has facilitated relaxation, breathing control and aided sleep

- POS

Its use has identified a gap in care when patients are not accessing other hospice services and ensured their needs are met.

Conclusion The exercise group has proved to be a good leveller with patients feeling confident to share experiences whilst gaining physical and emotional strength.

\section{P-141 'SMILE' DEVELOPING A SELF-MANAGEMENT PROGRAMME WITHIN A HOSPICE OUT-PATIENT SETTING}

Victoria O'Cuinneagain, Nicola Davies, Wendy Valentine. The Hospice of St Francis, Berkhamsted, UK

10.1136/bmjspcare-2017-hospice. 166

Background The hospice out-patient service offers support to patients, carers and the bereaved, we identified that clients presented with problems often poorly recognised or understood. These were significant in creating barriers and impacting on ability to self-manage and maintain independence.

Aim To educate and empower through the promotion of selfmanagement skills and coping strategies; using a series of interlinked workshops addressing commonalities of symptoms impacting on everyday living.

Method A rolling programme of workshops was designed, running bi-monthly entitled; 'SMILE'- Self- Management and Independent Living Everyday. The programme comprises four workshops;

- Fatigue management

- Sleep and relaxation

- Mindfulness and resilience

- Movement and exercise.

Results Each workshop is evaluated with positive feedback being obtained in all areas. The most notable being:

- The information and skills I have learnt have helped me now $-76 \%$ strongly agree

- The information and skills I have learnt will help me in the future $-80 \%$ strongly agree.

The Well-Being Star Outcome Measure is also used. Areas of particular influence noted were on 'symptom management', feeling positive', 'looking after yourself' and 'lifestyle'.

Feedback received:

- 'Realising sleep problems are very common and I am not alone!'

- 'giving me insight into multiple causes of fatigue'

- 'being given coping examples'.

Conclusion Patients have demonstrated a greater level of empowerment and self-management, seeking additional support. This has included requests for help returning to work, an area of support not previously in high demand. As a result of the positive outcomes there has been an increased referral rate. Attendance at sessions is variable with 'Fatigue Management' and 'Sleep and Relaxation' being most popular. The programme has been enhanced to offer individual sessions for those not suited to a group setting. Additionally increased promotion is required to encourage carers and bereaved people to recognise 'SMILE' is not a patient only programme.

\section{P-142 EIGHT POSITIVE STEPS TO WELLBEING...}

Sally List. Earl Mountbatten Hospice, Isle of Wight, UK

10.1136/bmjspcare-2017-hospice. 167

Background Self-management support can be viewed in two ways - as a portfolio of techniques and tools to help patients choose healthy behaviours, and as a fundamental transformation of the patient-caregiver relationship into a collaborative partnership. The hospice delivers a patient and carer programme to encourage self-management for patients with a range of life-limiting conditions. The planning of the programme was carried out with the input of the hospice User Forum, with the scoping of existing groups delivered by hospices across England. The first programme was initially piloted to identify the key sessions and format of the programme. Self-management programmes for patients with long-term conditions have been developed and researched over the past decade. There is limited research concerning these for adults with life-limiting conditions.

Aims Supporting adults with any life-limiting illness - offering information and support to help people manage their illness along with carers, family and friends who support them.

Methods People are invited to attend one or more sessions, which include eating well, living with fatigue, encouraging exercise, coping with being breathless, protecting skin, information about welfare benefits and how to cope with medical and care changes. The hour-long sessions are facilitated by an expert in the relevant field either from the hospice or partner organisations, including Age UK and the NHS Trust.

Results First programme - 31 attendees; second programme 53 attendees. Examples of participant feedback - 'I feel rejuvenated'; 'I close my flat door and come here for positive input'; 'I had no idea of the range of moulds for the pureed foods I need to have'.

Conclusions Voluntary participation, positive group dynamics, strong but flexible facilitators and active learning techniques underpin the overwhelmingly positive experiences of the sessions now being rolled out across the year.

\section{P-143 ENABLING NOT DISABLING}

Becky McGregor, Elizabeth Arnold. Earl Mountbatten Hospice, Isle of Wight, UK

10.1136/bmjspcare-2017-hospice. 168

Introduction A successful funding application to the St James's Place Foundation through Hospice UK has seen the commencement of a project focusing on enabling rather than disabling people; blending compassion and care with a rehabilitative and enabling approach.

Aims of the Project The project aims to shift the culture from one of 'over caring' within hospice services, to one that is working for and with people to determine what living well 